\title{
Superposition of local zoom models and simultaneous calibration for 1D-2D shallow water flows
}

\author{
J. Marin ${ }^{a}$ J. Monnier b,* \\ ${ }^{a}$ Univ. Grenoble 8 INRIA, Lab. LJK, B.P. 53, F-38041 Grenoble Cedex 9, France \\ ${ }^{\mathrm{b}}$ Univ. Toulouse - INSA, Institut de Mathématiques, F-31077 Toulouse cedex 4, \\ France
}

\section{Résumé}

We address the problem of coupling 2D Shallow Water equations with 1D Shallow Water equations (St-Venant equations), as applied to river-floodplain flows. Mathematical coupling conditions are derived classicaly from the 3D Navier-Stokes equations by integrating over the vertical wetsection, when overflowing occurs. It leads to extra source terms in the 1D equations. Next we assume to be in a variationnal data assimilation context, then the optimal control process allows to couple both models and assimilate data simultaneously (Joint-Assimilation-Coupling algorithms). Two different versions of JAC algorithms are presented and compared. In a numerical test case, we superimpose the local 2D model on the 1D global model. The results show the efficiency of the present simultaneous superposition - assimilation approach.

Key words: Shallow Water equations, Saint-Venant equation, coupling, zoom model, superposition, optimal control, data assimilation, river, flood plain. PACS:

\section{Introduction}

In river hydraulics, operational models are based on 1D Shallow Water equations with storage areas (also called 1.5D model) since 2D models cannot be solved for large scale in a reasonable CPU-time, see [4] or e.g. [20,18] for recent reviews. In 1.5D models, floodplains are modelled by storage areas (static volumes storing water). These models consist in 1D equations with extra source

*. Corresponding author : Jerome.Monnier@insa-toulouse.fr 
terms which need to be calibrated empirically. Obviously this type of model does not describe flow dynamics inside the storage area. For any reason, the end-user of river hydraulics models may have to model the flow inside the storage areas (e.g. flood plains). Furthermore, in a data assimilation context, one may have data available in the flooded areas (e.g. spatially distributed water levels extracted from a satellite image, see $[16,15])$. Obviously such data are not represented by the $1 \mathrm{D}$ model. A way to use such data is to superimpose a local 2D zoom model on the $1 \mathrm{D}$ global model and to perform a data assimilation process for the coupled model 1D-2D. This is the idea we develop in the present article. We address the mathematical problem of coupling the 1D Shallow Water equations to the 2D Shallow Water equations (superposition of a local 2D zoom model over the $1 \mathrm{D}$ global model), whereas numerically we couple both models using an optimal control process.

The coupling problem has already been addressed in few papers. Classicaly, the geometrical domain (and mesh) is decomposed, the 1D equations are coupled with the 2D ones via boundary conditions at interfaces, see e.g. $[20,17,10]$. An efficient coupling procedure may be a Schwarz-like algorithm, see e.g. [19]. In the present approach, we superimpose the 2D model on the 1D model. The coupling terms in the 1D equations are source terms, whereas in the 2D equations they are boundary conditions at open boundaries (of the $2 \mathrm{D}$ model). Then, we define an algorithm of coupling based on an optimal control process. Let us recall that the latter is already available if we suppose to be in a variationnal data assimilation context. Thus, we can superimpose both models and assimilate data simultaneously. Let us point out that the simultaneous assimilation allows to couple both models accurately since we do use data to define quantatively the coupling terms.

Shallow-water models (e.g. for river flows) require information on the value of input parameters (e.g. Manning coefficients), initial conditions and boundary conditions (e.g. inflow discharge in the main channel) in order to compute a flow state as reliable as possible, i.e. as close as possible to reality. To this purpose, data assimilation methods make it possible to combine optimally the equations of the model and observations. Variationnal data assimilation is based on the optimal control of the (forward) model. This has been applied successfully in river hydraulics, see e.g. [5,1,18,3,12,13,14,16,15].

Let us assume that we are in an variationnal data assimilation context : the model calibration is based on a optimal control process which includes the adjoint equations and a minimization procedure, see e.g. [12]. Then, we take advantage of the already existing optimal control process, in order to couple both models. In other words, we couple both models while at same time we 
assimilate data. This is the basic idea of the Joint Assimilation Coupling (JAC) algorithms we introduce in the present article. A preliminary approach has been introduced in [8]. We follow the same idea and we improve the method. The main improvements are the following. First we derive mathematically the coupling source terms appearing in the 1D equations from the 3D NavierStokes equations; second we consider the right characteristics variables at open boundaries of the 2D model (interfaces between both models); third we present an extra version of JAC algorithm, and we compare it to the first JAC algorithm introduced in [8].

An extra difficulty when coupling numerically arises from the unmatching grids (grids do not match both in space and time). Typical ratio of space and time grids between the $2 \mathrm{D}$ and the $1 \mathrm{D}$ are $\approx 10^{2}$ (the discrete $2 \mathrm{D}$ model being the finest one). These differences of grids can be easily handled when using the present optimal control approach.

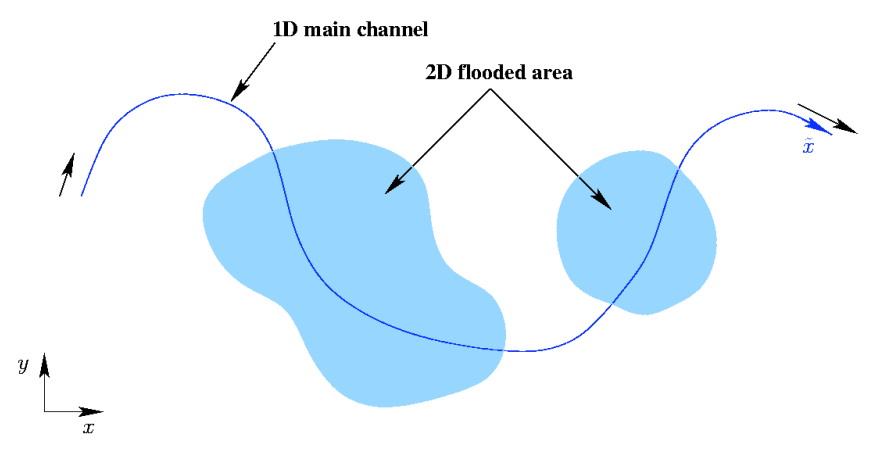

Figure 1. Modelling outline : a global 1D model with superposed local 2D models.

Outline of the paper is as follows. In Section 2, we derive the 1.5D model from the 3D Navier-Stokes equations, in particular the coupling source terms in the $1 \mathrm{D}$ equations. In other respect, the 2D Shallow Water equations are recalled. In Section 3, we focus on the coupling conditions between both models. In Section 4, we present the two Joint Assimilation Coupling (JAC) algorithms. In Section 5, we perform numerical tests. Coupling and data assimilation are done simultaneously using the two JAC type algorithms. We show the efficiency of both algorithms, and we discuss advantages and drawbacks of each algorithm. In Appendix A, we derive the expression of characteristics of the 2D model (conditions at open boundaries).

\section{Mathematical models}

\subsection{Derivation of the $1.5 D$ model}

The 1.5D model is based on the classical 1D Shallow-Water equations (or Saint-Venant equations). Nevertheless, since our goal is to couple the 1D equa- 
tions with the $2 \mathrm{D}$ equations, we must take into account transfers through the two lateral boundaries of the 1D main channel, see figures 1 and 2. To this end, we follow the classical derivation of 1D Saint-Venant equations, but in the derivation we keep the lateral transfer terms if overflowing at lateral boundaries. This leads to source terms in the 1D equations.

Let us consider the 3D incompressible Navier Stokes equations in the domain represented in Fig. 2. We impose no-slip boundary conditions at bottom and the kinematic equation at free surface.

We denote by $: h$ the water depth, $\mathbf{u}$ the velocity, $z_{b}$ the topography, and $z_{s}=\left(h+z_{b}\right)$ the water elevation. We assume that at its extremities, the 1D main channel is open (open boundaries), Fig. 1. Furthermore, local overflowing occurs (in flooded areas, it will be modeled by the $2 \mathrm{D}$ equations) but no overflowing occurs at the 1D channel extremities.

We denote by $b_{k}(x), k=1,2$, the $1 \mathrm{D}$ channel lateral boundaries, Fig. 2 . We denote by $\left(\mathbf{t}_{k}, \mathbf{n}_{k}\right), k=1,2$, the unit tangential - external normal vectors to the lateral boundary $k$. We have :

$\mathbf{n}_{1}=\frac{1}{\delta_{1}}\left(\partial_{x} b_{1},-1\right)^{T}$ and $\mathbf{n}_{2}=\frac{1}{\delta_{2}}\left(-\partial_{x} b_{2}, 1\right)^{T}$ with $\delta_{k}=\sqrt{1+\left(\partial_{x} b_{k}\right)^{2}}, \quad k=1,2$.

We denote by $S$ the wet cross section and by $Q$ the lineic-discharge in the main channel :

$$
S=\int_{b_{1}}^{b_{2}} \int_{z_{b}}^{z_{s}} d z d y \text { and } Q=\int_{b_{1}}^{b_{2}} \int_{z_{b}}^{z_{s}} u d z d y
$$

Mass conservation. Using standard notations, the mass conservation equation writes : $\frac{\partial u}{\partial x}+\frac{\partial v}{\partial y}+\frac{\partial w}{\partial z}=0$. By integration over $S$ we get :

$$
\underbrace{\int_{b_{1}}^{b_{2}} \int_{z_{b}}^{z_{s}} \frac{\partial u}{\partial x} d z d y}_{A}+\underbrace{\int_{b_{1}}^{b_{2}} \int_{z_{b}}^{z_{s}} \frac{\partial v}{\partial y} d z d y}_{B}+\underbrace{\int_{b_{1}}^{b_{2}} \int_{z_{b}}^{z_{s}} \frac{\partial w}{\partial z} d z d y}_{C}=0
$$

We can re-write expressions $A, B$ and $C$, using Leibniz's integration rule and 


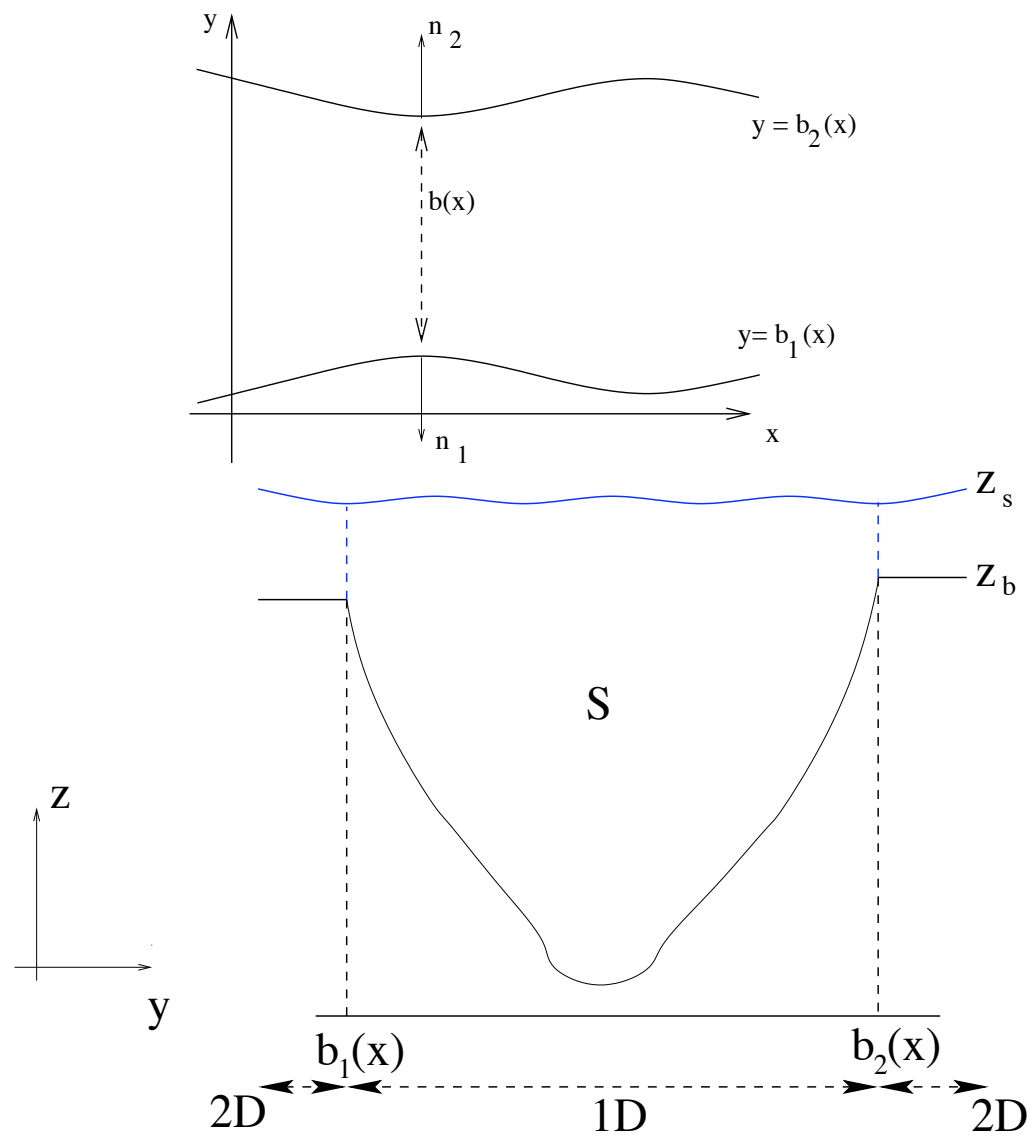

Figure 2. Configuration and notations. Up : Overview of the 1D channel. Down : $1 \mathrm{D}$ cross section with overflowing

the no-slip condition at the bottom :

$$
\begin{aligned}
A & =\int_{b_{1}}^{b_{2}}\left(\frac{\partial}{\partial x} \int_{z_{b}}^{z_{s}} u d z-u_{S} \frac{\partial z_{s}}{\partial x}\right) d y \\
& =\frac{\partial}{\partial x} \int_{b_{1}}^{b_{2}} \int_{z_{b}}^{z_{s}} u d z d y-\left[\int_{z_{b}}^{z_{s}} u d z\right]_{b_{2}} \frac{\partial b_{2}}{\partial x}+\left[\int_{z_{b}}^{z_{s}} u d z\right]_{b_{1}} \frac{\partial b_{1}}{\partial x}-\underbrace{\int_{b_{1}}^{b_{2}} u_{S} \frac{\partial z_{s}}{\partial x} d y}_{C L 1} \\
B & =\int_{b_{1}}^{b_{2}}\left(\frac{\partial}{\partial y} \int_{z_{b}}^{z_{s}} v d z-v_{S} \frac{\partial z_{s}}{\partial y}\right) d y \\
& =\left[\int_{z_{b}}^{z_{s}} v d z\right]_{b_{2}}-\left[\int_{z_{b}}^{z_{s}} v d z\right]_{b_{1}}-\underbrace{\int_{b_{1}}^{b_{2}} v_{S} \frac{\partial z_{s}}{\partial y} d y}_{C L 2} \\
C & =\underbrace{\int_{b_{1}}^{b_{2}} w_{S} d y}_{C L 3}
\end{aligned}
$$

In other respect, the free surface boundary condition gives :

$$
C L 1+C L 2+C L 3=\int_{b_{1}}^{b_{2}} \frac{\partial z_{s}}{\partial t} d y
$$


Thus, Equation (2) becomes :

$$
\frac{\partial S}{\partial t}+\frac{\partial Q}{\partial x}=K_{1}
$$

with :

$$
K_{1}=-\left\{\left[\int_{z_{b}}^{z_{s}} v d z\right]_{b_{2}}-\left[\int_{z_{b}}^{z_{s}} u d z\right]_{b_{2}} \frac{\partial b_{2}}{\partial x}-\left[\int_{z_{b}}^{z_{s}} v d z\right]_{b_{1}}+\left[\int_{z_{b}}^{z_{s}} u d z\right]_{b_{1}} \frac{\partial b_{1}}{\partial x}\right\}
$$

Without overflowing, at lateral boundaries (i.e. at $y=b_{k}, k=1,2$ ) we have $z_{s}=z_{b}$ and $K_{1}=0$. Thus, we obtain the first equation of the standard 1D St-Venant equations.

With overflowing and if coupling with a $2 \mathrm{D}$ model, $\left[\int_{z_{b}}^{z_{s}} u d z\right]_{b_{k}}$ and $\left[\int_{z_{b}}^{z_{s}} v d z\right]_{b_{k}}, k=$ 1,2 , represent the lineic-discharges on the two lateral boundaries in $x$-direction and $y$-direction respectively.

Let us introduce the mean discharge at lateral boundary $k$ :

$$
\overline{\mathbf{q}}_{k}=\left(\left[\int_{z_{b}}^{z_{s}} u d z\right]_{b_{k}},\left[\int_{z_{b}}^{z_{s}} v d z\right]_{b_{k}}\right) \quad k=1,2
$$

We set the normal mean discharge at lateral boundaries :

$$
q_{\mathbf{n}_{k}}=\overline{\mathbf{q}}_{k} \cdot \mathbf{n}_{k} \quad k=1,2
$$

Then we obtain : $K_{1}=-\delta_{1} q_{\mathbf{n}_{1}}-\delta_{2} q_{\mathbf{n}_{2}}$. If the canal width variation is small then $\delta_{k} \approx 1$ and : $K_{1} \approx-\left(q_{\mathbf{n}_{1}}+q_{\mathbf{n}_{2}}\right)$.

Let us assume that the canal width variation is small $\left(\delta_{k} \approx 1\right)$. Then the mass conservation equation becomes :

$$
\frac{\partial S}{\partial t}+\frac{\partial Q}{\partial x}=-\left(q_{\mathbf{n}_{1}}+q_{\mathbf{n}_{2}}\right)
$$

The values $q_{\mathbf{n}_{k}}, k=1,2$, have to be provided by the $2 \mathrm{D}$ model.

Momentum conservation. Using standard notations, we consider the following 3D Navier-Stokes momentum equation : $\frac{\partial u}{\partial t}+u \frac{\partial u}{\partial x}+v \frac{\partial u}{\partial y}+w \frac{\partial u}{\partial z}+\frac{1}{\rho} \frac{\partial p}{\partial x}=\nu \Delta u$. Using the incompressibility condition, this gives: $\frac{\partial u}{\partial t}+\frac{\partial u^{2}}{\partial x}+\frac{\partial u v}{\partial y}+\frac{\partial u w}{\partial z}+\frac{1}{\rho} \frac{\partial p}{\partial x}=$ $\nu \Delta u$.

As previously, we integrate the equation over the wet cross section S. Again, we assume that the canal width variation is small. We denote by $H$ the mean 
transverse water depth and by $\mathcal{Z}_{b}$ the mean transverse topography. We consider the following hydrostatic pressure $: p=-\rho g\left(z-\left(H+\mathcal{Z}_{b}\right)\right)$. We use the boundary conditions at bottom and free surface, we use the Leibniz's integration rule. We obtain :

$$
\frac{\partial Q}{\partial t}+\frac{\partial}{\partial x}\left(\beta \frac{Q^{2}}{S}\right)+g S \frac{\partial\left(H+\mathcal{Z}_{b}\right)}{\partial x}=K_{2}-g S S_{f}
$$

where the nonlinear friction term $S_{f}$, deriving from the viscous term, is modelled using the classical Manning-Strickler's law. The Boussinesq coefficient $\beta$ is defined by :

$$
\beta=\frac{S}{Q^{2}} \int_{b_{1}}^{b_{2}} \int_{z_{b}}^{z_{s}} u^{2} d z d y
$$

Let us remark that if $u$ is constant over the cross section $S$ then $\beta=1$.

The source term $K_{2}$ is :

$$
K_{2}=-\left[\int_{z_{b}}^{z_{s}} u v d z\right]_{b_{2}}+\left[\int_{z_{b}}^{z_{s}} u v d z\right]_{b_{1}}-\left[\int_{z_{b}}^{z_{s}} u^{2} d z\right]_{b_{1}} \frac{\partial b_{1}}{\partial x}+\left[\int_{z_{b}}^{z_{s}} u^{2} d z\right]_{b_{2}} \frac{\partial b_{2}}{\partial x}
$$

Let us point out that without overflowing $z_{s}=z_{b}$ at lateral boundaries, the term $K_{2}$ vanishes, and we obtain the standard 1D St-Venant equations.

Let us introduce the following two coefficients :

$$
\gamma_{k}=\frac{\left[\int_{z_{b}}^{z_{s}} u v d z\right]_{b_{k}}}{\left[\int_{z_{b}}^{z_{s}} u d z\right]_{b_{k}}\left[\int_{z_{b}}^{z_{s}} v d z\right]_{b_{k}}}, \quad \alpha_{k}=\frac{\left[\int_{z_{b}}^{z_{s}} u^{2} d z\right]_{b_{k}}}{\left(\left[\int_{z_{b}}^{z_{s}} u d z\right]_{b_{k}}\right)^{2}}, \quad k=1,2
$$

If $(u, v)$ does not depend on $z$ on lateral boundary $b_{k}, k=1,2$, then $\gamma_{k}=$ $\alpha_{k}=1 / h_{k}$.

Let us assume that : $\beta=\delta_{k}=1, k=1,2$, and $(u, v)$ does not depend on $z$ on lateral boundaries. Then we obtain the following shallow momentum equation :

$$
\frac{\partial Q}{\partial t}+\frac{\partial}{\partial x}\left(\frac{Q^{2}}{S}\right)+g S \frac{\partial\left(H+\mathcal{Z}_{b}\right)}{\partial x}=-\left(\left.u\right|_{1} q_{\mathbf{n}_{1}}+\left.u\right|_{2} q_{\mathbf{n}_{2}}\right)-g S S_{f}
$$

Let us remark that if no 2D equations are available to model the overflowing, then one can set $\left.u\right|_{k}=\frac{Q}{S}, k=1,2$, at lateral boundaries and we obtain get the classic so-called "1D model with storage area" (also called 1.5D model), see e.g. [4], [18]. 
Rectangular cross-section case. For the sake of simplicity, we consider rectangular cross sections in the main channel, then : $S=b H$, where $b$ is the channel width. If we define the pressure term, see e.g. [9] :

$$
P=g S \frac{H}{2}=g \frac{S^{2}}{2 b}
$$

then we have $: \frac{\partial P}{\partial x}=g\left(\frac{\partial b}{\partial x} \frac{H^{2}}{2}+S \frac{\partial H}{\partial x}\right)$.

Finally the equations (5) and (8) can be written as an hyperbolic system with source terms as follows :

$$
\left\{\begin{array}{l}
\frac{\partial S}{\partial t}+\frac{\partial Q}{\partial x}=-\left(q_{\mathbf{n}_{1}}+q_{\mathbf{n}_{2}}\right) \\
\frac{\partial Q}{\partial t}+\frac{\partial}{\partial x}\left(\frac{Q^{2}}{S}+P\right)-g \frac{\partial b}{\partial x} \frac{H^{2}}{2}+g S \frac{\partial \mathcal{Z}_{b}}{\partial x}=-\left(\left.q_{\mathbf{n}_{1}} u\right|_{1}+\left.q_{\mathbf{n}_{2}} u\right|_{2}\right)-g S S_{f}
\end{array}\right.
$$

The source term

$$
\Psi=\left(-\left(q_{\mathbf{n}_{1}}+q_{\mathbf{n}_{2}}\right),-\left(q_{\mathbf{n}_{1}} u_{\mathbf{t}_{1}}+q_{\mathbf{n}_{2}} u_{\mathbf{t}_{2}}\right)\right)
$$

is the coupling term with the $2 \mathrm{D}$ equations.

\subsection{The 2D model}

The 2D hydraulics model is based on the classical bidimensional ShallowWater equations in their conservative formulation. The unknowns are the water depth $h$ and the local discharge $\mathbf{q}=h \mathbf{u}=\left(q_{x}, q_{y}\right)$, where $\mathbf{u}=(u, v)^{T}$ is the depth-averaged velocity vector. Equations are :

$$
\left\{\begin{array}{l}
\partial_{t} h+\partial_{x} q_{x}+\partial_{y} q_{y}=0 \\
\partial_{t} q_{x}+\partial_{x}\left(\frac{q_{x}^{2}}{2}+\frac{1}{2} g h^{2}\right)+\partial_{y}\left(\frac{q_{x} q_{y}}{h}\right)=-g\left[h \partial_{x} z_{b}+\frac{n^{2}\|\mathbf{q}\|_{2}}{h^{7 / 3}} q_{x}\right] \\
\partial_{t} q_{y}+\partial_{x}\left(\frac{q_{x} q_{y}}{h}\right)+\partial_{y}\left(\frac{q_{y}^{2}}{2}+\frac{1}{2} g h^{2}\right)=-g\left[h \partial_{y} z_{b}+\frac{n^{2}\|\mathbf{q}\|_{2}}{h^{7 / 3}} q_{y}\right]
\end{array}\right.
$$

where $z_{b}$ is the bed elevation and $n$ is the Manning roughness coefficient. The system is closed with appropriate boundary conditions (see later) and initial conditions $\left(h_{0}, q_{0}\right)$. As previously, the system can be written as a $2 \mathrm{D}$ hyperbolic system with source terms; the source terms are the friction term plus the topography term (right hand-sides of Equation (11)). 


\section{Coupling conditions between the two models}

We focus on the interactions between the two models $1.5 \mathrm{D}$ and 2D. These interactions are bilateral : from the $1.5 \mathrm{D}$ model to the $2 \mathrm{D}$ model, and from the $2 \mathrm{D}$ model to the $1.5 \mathrm{D}$ model.

The information exchange from the $1.5 \mathrm{D}$ model to the $2 \mathrm{D}$ model is done through the boundary conditions of 2D model (at its open boundaries). Since it is an open boundary condition problem, since the equations are hyperbolic, it is natural to introduce characteristic variables [2] whose definitions are detailed in Appendix A. In short, at interfaces we impose that incoming characteristics in $2 \mathrm{D}$ model equal those computed by the $1.5 \mathrm{D}$ model (see Fig. 3 ) :

$$
\int_{\Gamma_{l}} w_{i} d s=W_{i}^{\Gamma_{l}} ; l \in\{\text { in }, \text { out }\}, i=1,2
$$

The information exchange from the $2 \mathrm{D}$ model to the $1.5 \mathrm{D}$ model is done via the source term $\Psi$ defined previously, see (10).

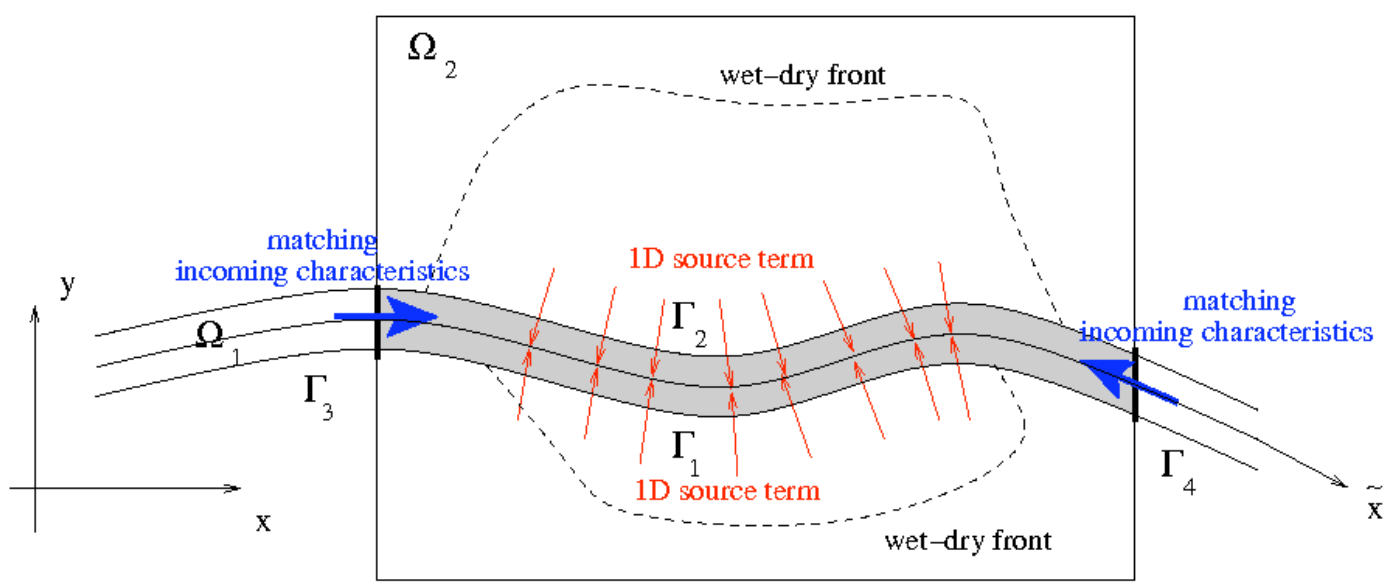

FiguRE 3. Information transfer. 1D main channel $\Omega_{1}$, 2D flood plain $\Omega_{2}$, overflowing boundaries $\Gamma_{1}, \Gamma_{2}$

\section{The Joint-Assimilation-Coupling (JAC) algorithms}

If the goal is to couple both models only, different coupling algorithms can be considered. One can consider the most classical one : a Schwarz-like algorithm, those global in time for example, see e.g. [19]. We have tested such an approach, see [7] for details, and this leads to satisfying results : after few iterations only, both models are coupled in an efficient way. Therefore, if forward modelling only is required, a Schwarz algorithm global in time is a simple and reliable approach to couple both models. In case of inconsistant grids (in space and time) between the $1.5 \mathrm{D}$ and the $2 \mathrm{D}$ models, one can use efficient 
interpolation procedures to transfer information between both models.

Now, let us assume that we are in a calibration context based on variational data assimilation, see e.g. [11] [12]. That suppose a whole optimal control process based on adjoint equations and a minimization algorithm is already implemented. In that case, one can take advantage of the existing optimal control process to couple both models simultaneously. In other words, one can couple both models while at same time we assimilate data into the coupled model. In addition, the assimilation allows to couple both models accurately since we do use data to define quantatively the coupling terms.

First, we recall the variational data assimilation principles. Then, we consider the JAC algorithm introduced [8], but here we use it with the coupling conditions derived rigorously in Section 2. Next, we introduce a new version of JAC algorithm (the "sequential version"). Also, we compare both algorithms, we discuss their respective advantages and drawbacks.

\subsection{Variationnal data assimilation principles}

Variationnal data assimilation consists to make fit in an optimal way the computed state with observations. This is done by minimizing a cost function measuring the discrepancy between both. The local minimization procedure requires the gradient of cost function, it is obtained by solving adjoint equations, see e.g. $[12,13,14,16,15]$. If we denote by $k$ the control variable (in floodplain flows, it can be for example inflow discharge or Manning roughness coefficients), the optimization problem is :

$$
\min _{k} J(k)
$$

where $J(k)=G\left(k ; \mathbf{y}^{k}\right), \mathbf{y}^{\mathbf{k}}$ is the state of the system at $k$ given. The cost function is generally in the following form :

$$
J(k)=J_{o b s}(k)+J_{\text {reg }}(k)
$$

where the cost function term $J_{o b s}$ is defined by : $J_{o b s}=\left\|\mathbf{y}-\mathbf{y}^{o b s}\right\|_{*}^{2}$. It measures the discrepancy between the computed state $\mathbf{y}$ and observations $\mathbf{y}^{\text {obs }}$ in a given norm $\|\cdot\|_{*}$.

The regularization term $J_{\text {reg }}$ (Tikhonov's regularization) is very usefull on one hand to "convexify" the cost function, on the other hand to smooth the control variable. As an example, if the control variable is the inflow discharge $k=Q_{\text {in }}(t)$ (like in the numerical tests in next section), one can set : $J_{\text {reg }}=$ $\frac{1}{2}\left|\frac{\partial Q_{i n}}{\partial t}\right|^{2}$. 


\subsection{Joint Assimilation Coupling (JAC) type algorithms}

The relaxed $J A C$ algorithm. The $\mathrm{JAC}$ algorithm originally introduced in [8] is as follows, see Fig. 4. We relax the dependency between the 1.5D and the 2D model and we control the resulting weakly coupled model. In others words, in addition of classical control variables (here, inflow discharge), we control the $2 \mathrm{D}$ incoming characteristics at the $2 \mathrm{D}$ open boundaries. In other respects, we add the following term to the cost function :

$$
J_{\text {coupling }}(k)=\int_{0}^{T}\left[\sum_{l \in\{\text { in,out }\}} W_{i}^{\Gamma_{l}}-\int_{\Gamma_{l}} w_{i} d s\right] d t \quad ; i=1,2
$$

Then the total cost function we minimize becomes :

$$
J_{\text {tot }}(k)=\alpha_{1 D} J_{1 D}(k)+\alpha_{2 D} J_{2 D}(k)+\alpha_{\text {coupling }} J_{\text {coupling }}(k)+\alpha_{\text {reg }} J_{\text {reg }}(k)
$$

where $J_{1 D}$ and $J_{2 D}$ are classical cost function terms in data assimilation (as described above). The $\alpha_{\square}$ coefficients are setted "at best" as it is usualy done in multi-objective optimization i.e. they are valued in order to balance at best all terms after convergence.

As we show it in next section, the resulting "relaxed JAC algorithm" (Fig. 4) is efficient and quite robust. On the other hand, its drawbacks are the following: i) tunning the $\alpha_{*}$ coefficients can be awkward (it is a multi-objective optimization problem)

ii) it requires the adjoint code of the "weakly coupled model". It is not very difficult to obtain from each adjoint codes but not straightforward.

The sequential JAC algorithm. Now, we define a sequential version of JAC algorithm. The basic idea is to control both models in a sequential way, see Fig. 5. The resulting "sequential JAC algorithm" presents the following three advantages :

i) it requires the adjoint codes of the two (physical) forward models separatively (and not the adjoint code of an unphysical forward model)

ii) the optimization problem related to the $2 \mathrm{D}$ model is a classical variational data assimilation problem. It does not make define extra cost function terms, thus it is not a more complex multi-objective optimization problem. Furthermore, this remark remains true at second stage (optimization problem related to the $1.5 \mathrm{D}$ model) if data are available in flood plain only (like it is the case in forthcoming numerical results)

iii) the two optimization problems are solved sequentially, therefore separatively.

Nevertheless, as we will show it in next section, this algorithm present a drawback when identifying inflow boundary conditions for example, since it requires 
a special treatment of the so-called "blind period" (see next section).

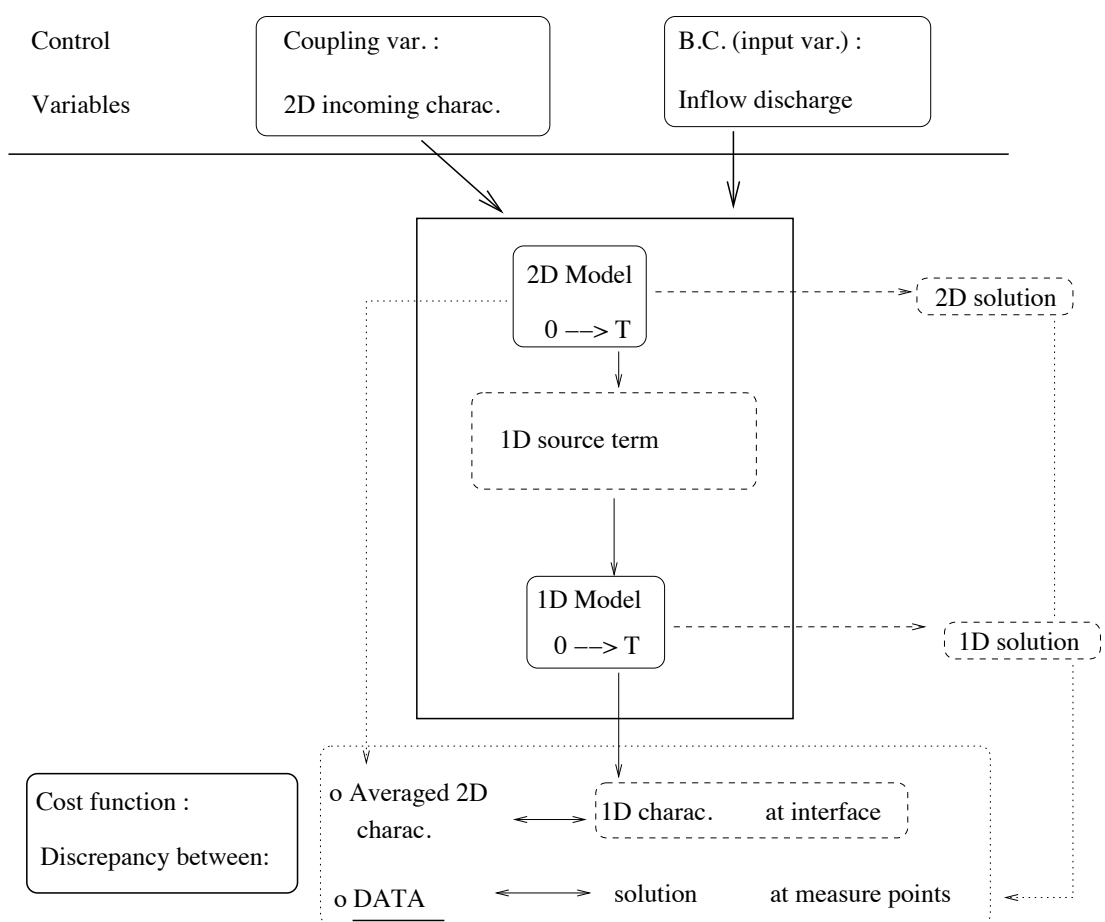

Figure 4. The relaxed JAC algorithm

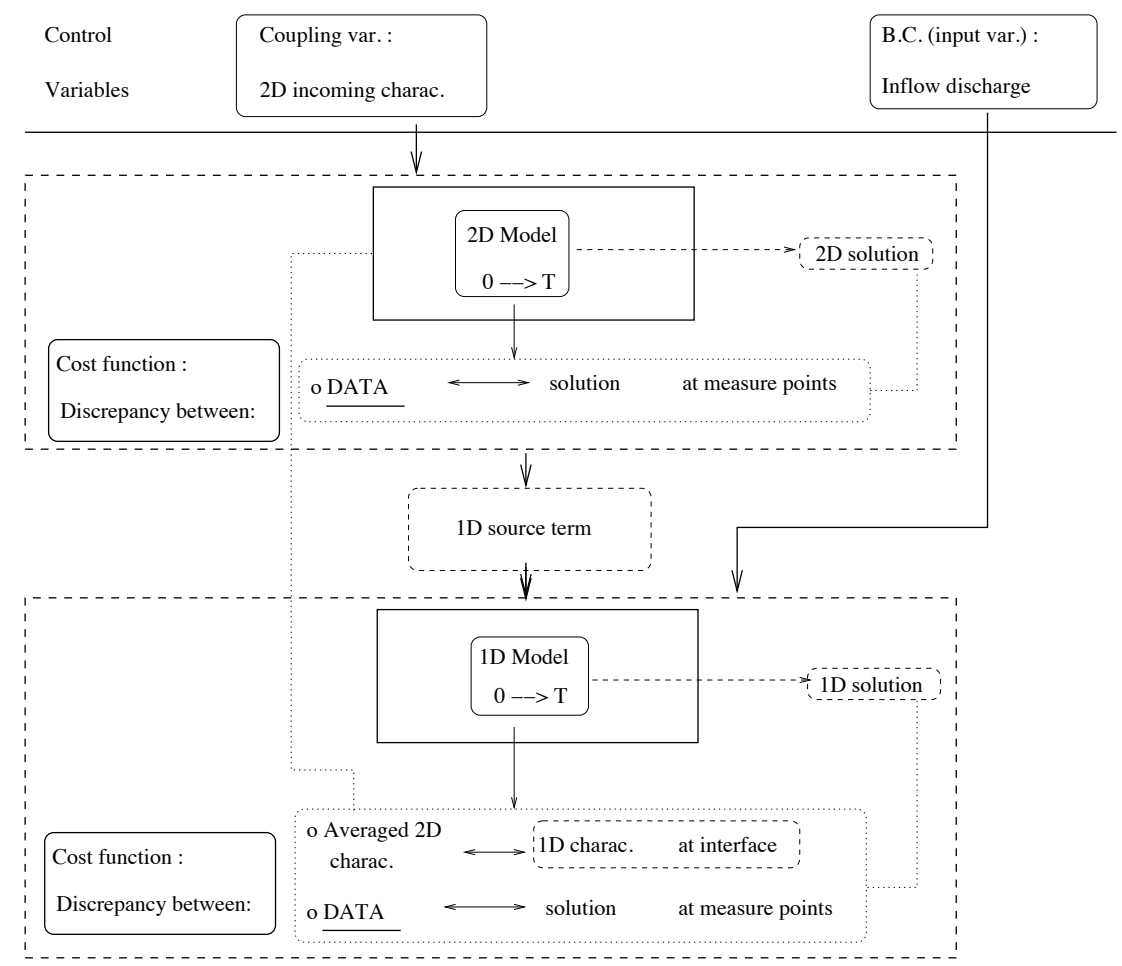

Figure 5. The sequential JAC algorithm 


\section{$5 \quad$ Numerical tests}

We seek to test and compare numerically the two JAC algorithms presented previously. To this end, we perform twin experiments. That means a preliminary run of the forward model provides observations. Next, input variables are changed, and we set them to a priori values ("first guesses"). Finally, the inverse problem consists to retrieve the "reference" input variables values. The numerical tests are performed using our software DassFlow $[12,11]$. It a computational software for free surface flows, including a variationnal data assimilation process. Both models (1.5D and 2D) are solved using well balanced finite volume schemes (HLL and HLLC respectively), explicit in time. We refer to $[12,6]$ for more details.

Test case description. The computational domain contains a main channel and a floodplain, see Fig. 6 a). The main channel is a straight-line (length $200 \mathrm{~m}$ ) with a flat bottom, and the 2D flood plain is a disk (diameter $100 \mathrm{~m}$ ) centered in the domain. Because of the dynamic wet/dry front (flood event), we do not consider a flat bottom in the $2 \mathrm{D}$ flood plain. Thus, we consider the virtually realistic bathymetry represented in Fig. 6 a).

The mesh is unstructured and contains 1400 cells. Boundary conditions at the 1D channel extremities are as follows. An hydrograph is prescribed at inflow boundary (see the reference curve in Fig. 7 a)), while homogeneous Neumann conditions are imposed at outflow boundary.

Let us point out that the solution of the forward coupled model (1D in the main channel coupled with the $2 \mathrm{D}$ in the flooded area) is exactly the same as the full 2D solution (difference in $L^{\infty}$-norm is about $10^{-7}$ ). A detailed study of the present numerical coupled model is done in [7].

The identification problem (inverse problem) we consider is the following. Given water height measurements at two gauging stations located in the $2 \mathrm{D}$ flood plain, see Fig. 6 a) and b), we seek to identify the 1D inflow hydrograph. Let us recall that the present tests are twin experiments, thus the measurements have been created using the model with the "right" 1D inflow hydrograph (i.e. the 1D inflow hydrograph we seek to retreive in the inverse problem).

Let us notice that the data we assimilate can be represented by the $2 \mathrm{D}$ model only (they would not be assimilable by a 1D model). Thus, we do assimilate $2 \mathrm{D}$ "local" data in order to calibrate / identify input variables of the 1D "global" model. 
Concerning the CPU times of JAC algorithms, they are both similar to the CPU-time of the data assimilation process for one model only (for example if using the $2 \mathrm{D}$ model only). It means that the extra coupling feature of the algorithms does not affect significantely the convergence speed of the minimzation process. Let us recall that the order of magnitude of a variational data assimilation process (4D-var) of 100 iterations (see Fig. 7 b) is about 400 times the cost of one run of the forward model.

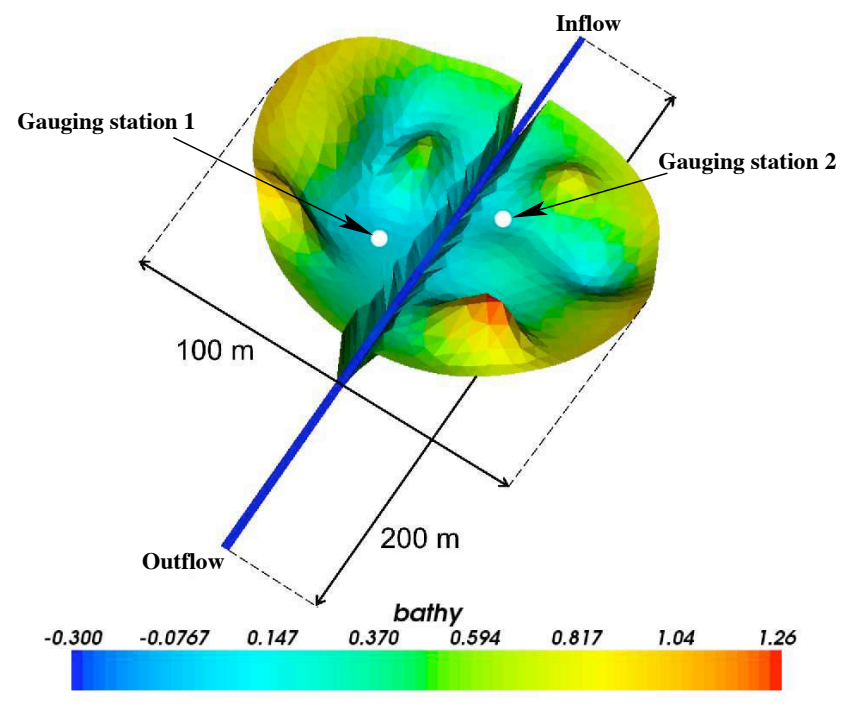

a)
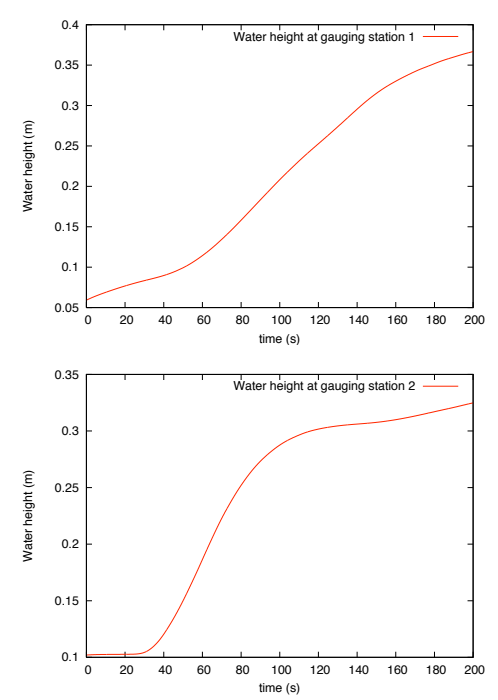

b)

Figure 6. a) Bathymetry. b) Observations : water height at two "gauging stations" in flood plain.

The relaxed JAC algorithm. We consider the algorithm presented in Fig. 4. The total cost function to minimize is given by (14). In the present test case, we have $J_{1 D}=0$ because no observation is available in the $1 \mathrm{D}$ channel. As mentionned previously, since the problem is multi-objective optimization problem, one must pay a particular attention to the choice of the ratio $\frac{\alpha_{\text {coupling }}}{\alpha_{2 D}}$. As a matter of fact, this choice may influence the convergence process. Here, choosing $\frac{\alpha_{\text {coupling }}}{\alpha_{2 D}}=0.001$, all terms of the cost function minimized are "wellbalanced" after convergence. As a first guess for the control variable (the inflow discharge), we choose a constant value equals to $6 \mathrm{~m}^{3} / \mathrm{s}$, see the green curve in Fig. 7 a). The results obtained are presented in Fig. 7 . After convergence, one can remark the perfect recovery of the 1D inflow hydrograph and incoming characteristics with the corresponding reference solution (see the blue and red curves in Fig. 7 a), c) and d)).

\section{Blind period phenomena.}

Let us remark that a blind period phenomena prevents us to identify boundary conditions at the end of the time interval. This blind period corresponds to 
the time required by the information to travel from the inflow boundary to the nearest observation point. Using a mean wave speed value $(u+\sqrt{g h})$, one can estimate roughly this blind period to $12 \mathrm{~s}$. Of course, one cannot identify the inflow discharge during this lap of time since its value does not affect the observations.

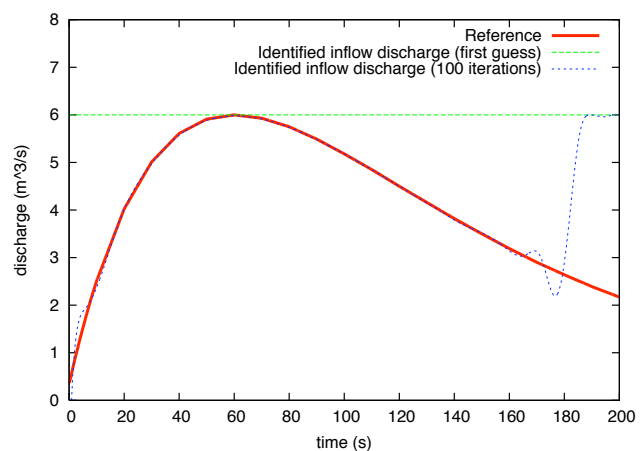

a)

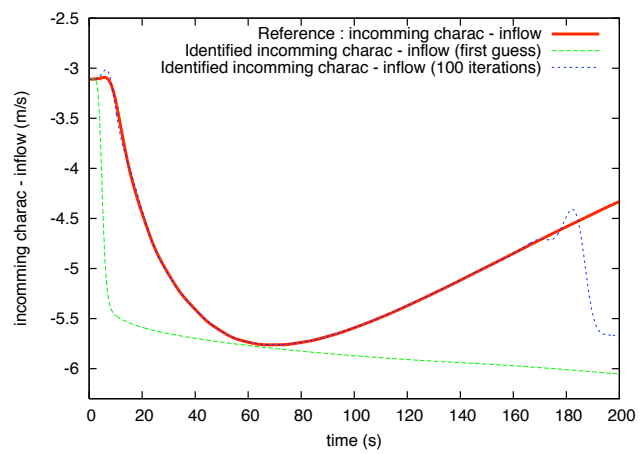

c)

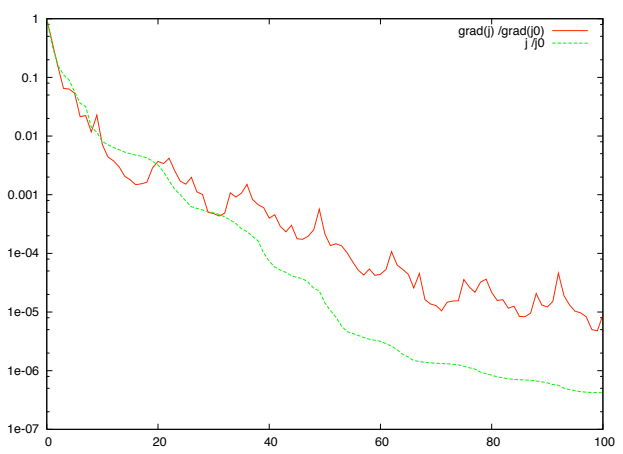

b)

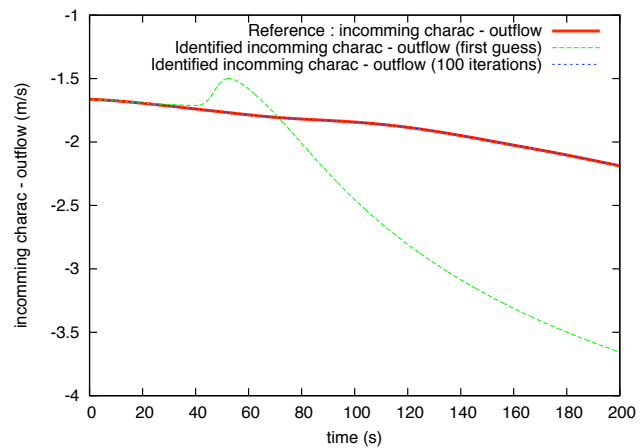

d)

FiguRE 7. Relaxed JAC algorithm : a) Identification of inflow hydrograph. b) Convergence history of the cost function. c) Identification of inflow incoming characteristic. d) Identification of outflow incoming characteristic.

The sequential JAC algorithm. We consider the algorithm presented in Fig. 5.

First step of the algorithm is the identification of 2D incoming characteristic variables. The forward model is the $2 \mathrm{D}$ model only. Given a first guess for incoming characteristics at open boundaries, we perform a full identification process which gives after convergence, optimal values for the incoming characteristics. The results obtained are presented in Fig. 8 a) and b). They show an excellent recovery of the identified 2D incoming characteristics with the reference ones (red curves). Of course, we observe the same blind period phenomena as before. That is why, one can consider that the identified incoming characteristics are accurate until a time $t^{*}$ (here $t^{*} \approx 160 \mathrm{~s}$ ), whereas one cannot identify the incoming characteristics in the time interval $\left[t^{*}, T\right]$.

After the first step, one can compute the "calibrated" source term $\Psi$ in the $1 \mathrm{D}$ equations. 
Second step is the identification of the 1D inflow discharge, given the calibrated source term $\Psi$. The forward model is the 1D model only. Since no 1D observation is available, we minimize the $J_{\text {coupling }}$ term only (plus a standard regularization term), see Equation (13), which measures the discrepancies of inflow characteristics at interfaces. The results obtained are presented in Fig. $8 \mathrm{c}$ ). Again, we obtain a very good recovery of the identified inflow discharge with the reference one (red curve). The convergence of the algorithm is presented in Fig. 8 d).

\section{Blind period phenomena.}

Because of the blind period phenomena in the first step, the second step may fail if we considere the identified incoming characteristics during the whole time interval $[0, T]$. As a matter of fact, since the adjoint equations propagate the information reverse in time, the variationnal data assimilation process would propagate the error at all time. Hence, before performing the second step we have to skip the degenerated time period in the $J_{\text {coupling }}$ term. It means we integrate in the time interval $\left[0, t^{*}\right]$ only, instead of $[0, T]$. Then, the $1 \mathrm{D}$ hydrograph is very well retrieved, see Fig. 8 c), excepted of course after the critical time $t^{*}$.

In summary, these two numerical tests show that both JAC algorithms allow to : a) couple accurately the 1D and 2D equations ; b) calibrate the 1D "global" model using observations in the 2D local zoom model.

The relaxed $J A C$ algorithm is efficient and robust but it is sensitive to the weight coefficients values and requires the derivation of an unphysical adjoint code. The sequential JAC algorithm has the great advantage to separate the two optimization processes (based each of them on the "standard" adjoint code). They can be performed sequentially.

Concerning the incontrovertible blind period, in the sequential JAC algorithm, one have to truncate "by hand" the assimilation time interval at second step. If not, the second identification process (4D-var algorithm for the $1 \mathrm{D}$ model) propagates the error at all time step and the minimization process fail to converge (to the right solution at least).

\section{Conclusion}

In this paper, we have coupled mathematically and numerically the $2 \mathrm{D}$ shallowwater equations (SWE) in $(h, q)$ variables with the $1 \mathrm{D}$ SWE in $(S, Q)$ variables, when overflowing occurs. Instead of a domain decomposition approach, we followed a superposition approach. The coupling term in 1D equations are source 


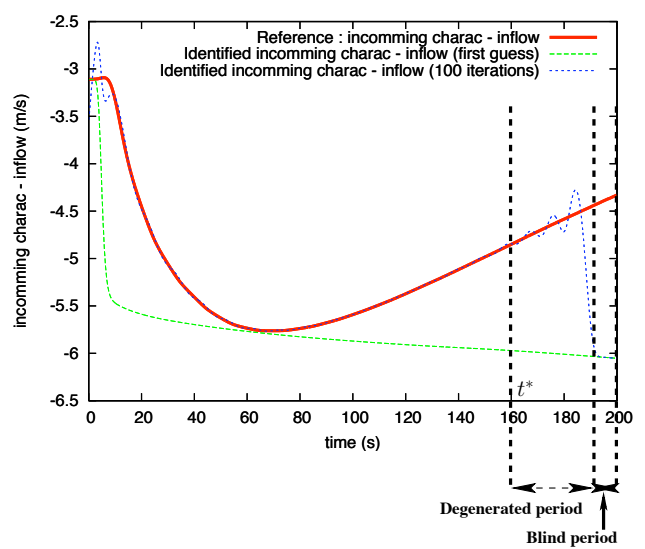

a)

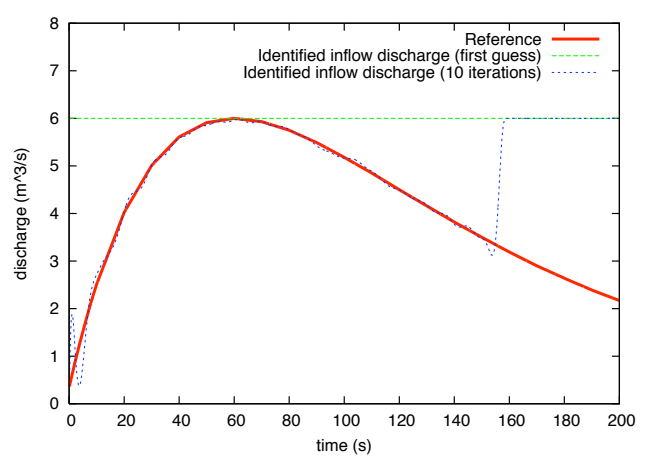

c)

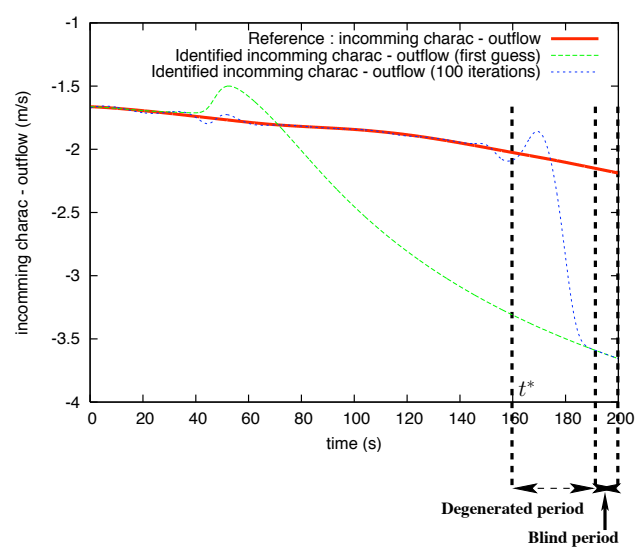

b)

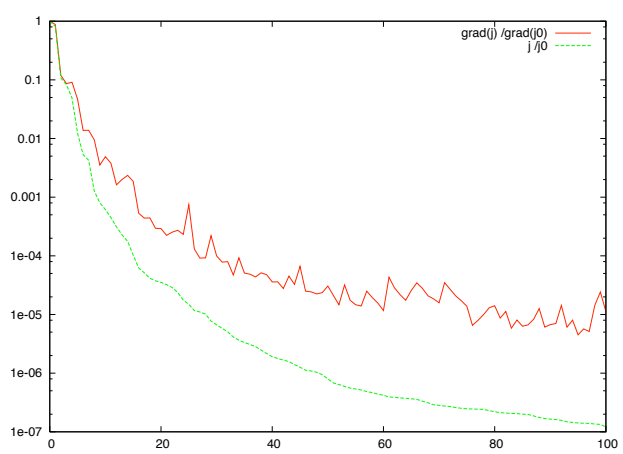

d)

FiguRE 8. Sequential JAC algorithm. First step : Idendification of incoming characteristic at inflow (a) and at outflow (b). Second step : c) Identification of inflow hydrograph. d) Convergence history of cost function.

terms derived from the 3D Navier-Stokes equations (similarly to 1D SWE with storage areas). The coupling terms in the $2 \mathrm{D}$ equations are the incoming characteristics at open boundaries. The coupling algorithm is based on an optimal control process. As a matter of fact, we assumed to be in a variational data assimilation context (4D-var algorithm), thus an optimal control loop (including adjoint equations and a minimization algorithm) is supposed to be available. Then, we can assimilate data and superimpose both models simultaneously. That approach presents few advantages. First, data enable to calibrate the model as it is done classicaly, but also enable to quantify the information transmitted between both models (i.e. to quantify the coupling terms). Second, if one has 2D data only (in the flood plain for example), the superposition of the 2D "local" model over the 1D one combined with the assimilation process, allows to calibrate the 1D "global" model. We present two Joint-Assimilation-Coupling (JAC) algorithms. Both present advantages and drawbacks. Numerical tests on an academic configuration are performed. They show the relevancy and the accuracy of these two JAC algorithms. Finally, the present approach (coupling/superposition and simultaneous data 
assimilation) can be naturally extended to the 3D Navier-Stokes equations 1D SWE system.

\section{A Characteristics boundary conditions}

In this section, we detail how we define open boundary conditions for $2 \mathrm{D}$ models. It is based on the theory of characteristics, see e.g. [21,2].

Let us consider the 2D SW equations near the boundary, in the non-conservative form and linearized around a mean value $\left(h_{0}, u_{0}, v_{0}\right)$, with a flat bottom and without friction :

$$
\left\{\begin{array}{l}
\frac{\partial u}{\partial t}+u_{0} \frac{\partial u}{\partial x}+v_{0} \frac{\partial u}{\partial y} g \frac{\partial h}{\partial x}=0 \\
\frac{\partial v}{\partial t}+u_{0} \frac{\partial v}{\partial x}+v_{0} \frac{\partial v}{\partial y}+g \frac{\partial h}{\partial y}=0 \\
\frac{\partial h}{\partial t}+u_{0} \frac{\partial h}{\partial x}+v_{0} \frac{\partial h}{\partial y}+h_{0}\left(\frac{\partial u}{\partial x}+\frac{\partial v}{\partial y}\right)=0
\end{array}\right.
$$

In a matrix form, this gives :

$$
U_{t}+A_{1} U_{x}+A_{2} U_{y}=0
$$

with $U=[h, u, v]^{T}, A_{1}=\left(u_{0} 0 h_{0}, 0 u_{0} 0, g 0 u_{0}\right)^{T}$ and $A_{2}=\left(v_{0} 00,0 v_{0} h_{0}, 0 g v_{0}\right)^{T}$ Let $\mathbf{n}=\left[n_{1}, n_{2}\right]^{T}$ and $\tau$ be respectively the normal and the tangent vector to the boundary. The matrix $A=n_{1} A_{1}+n_{2} A_{2}$ has 3 eigenvectors : $w_{1}=\mathbf{u} \cdot \mathbf{n}+\sqrt{\frac{g}{h_{0}}} h, w_{2}=\mathbf{u} \cdot \tau$ and $w_{3}=\mathbf{u} \cdot \mathbf{n}-\sqrt{\frac{g}{h_{0}}} h$. They are associated to the eigenvalues $\lambda_{1}=\mathbf{u}_{0} \cdot \mathbf{n}+c, \lambda_{2}=\mathbf{u}_{0} \cdot \tau$ and $\lambda_{3}=\mathbf{u}_{0} \cdot \mathbf{n}-c\left(c=\sqrt{g h_{0}}\right)$ respectively. These eigenvectors $w_{1}, w_{2}$ and $w_{3}$ are the so-called characteristic variables.

We rewrite (A.2), using $w_{1}, w_{2}$ and $w_{3}$ :

$$
\left\{\begin{array}{l}
\frac{\partial w_{3}}{\partial t}+\lambda_{3} \frac{\partial w_{3}}{\partial x_{\mathbf{n}}}+\mathbf{u}_{0} \cdot \tau \frac{\partial w_{3}}{\partial x_{\tau}}-c \frac{\partial v}{\partial x_{\tau}}=0 \\
\frac{\partial w_{2}}{\partial t}+\lambda_{2} \frac{\partial w_{2}}{\partial x_{\mathbf{n}}}+\mathbf{u}_{0} \cdot \tau \frac{\partial w_{2}}{\partial x_{\tau}}+\frac{c}{2} \frac{\partial\left(w_{1}-w_{3}\right)}{\partial x_{\tau}}=0 \\
\frac{\partial w_{1}}{\partial t}+\lambda_{1} \frac{\partial w_{1}}{\partial x_{\mathbf{n}}}+\mathbf{u}_{0} \cdot \tau \frac{\partial w_{1}}{\partial x_{\tau}}-c \frac{\partial v}{\partial x_{\tau}}=0
\end{array}\right.
$$

If we neglect the variations along $x_{\tau}$, (A.3) becomes a system of transport equations of $w_{k}$ at speed $\lambda_{k}$ in the normal direction $\mathbf{n}$. Given an open boundary, $w_{k}$ is incoming if $\lambda_{k}<0$ and outgoing otherwise.

Following [2], relevant open boundary conditions can be defined by imposing only the incoming characteristic variables on each boundary. 
Acknowledgments. The authors would like to thank Prof. E. Blayo (Lab. LJK, univ. Grenoble) for fruitful discussions concerning conditions at open boundaries, Dr J.-B. Faure (Cemagref Lyon) who helped to set the hydraulic problem. Also, the authors would like to thank a reviewer for his detailed comments that has helped to improve the redaction.

\section{Références}

[1] E. Belanger, A. Vincent. Data assimilation (4D-VAR) to forecast flood in shallow-waters with sediment erosion. J. Hydro. 300, pp. 114-125. 2005.

[2] E. Blayo, L. Debreu. Revisiting open boundary conditions from the point of view of characteristic variables. Ocean Modelling, vol 9, pp 231-252, 2005.

[3] W. Castaings, D. Dartus, M. Honnorat, F.-X. Le Dimet, Y. Loukili, J. Monnier. Automatic Differenciation : a tool for variational data assimilation and adjoint sensitivity analysis for flood modeling. Lecture Notes in Computational Science and Engineering. 50, pp. 249-262, 2006.

[4] J. A. Cunge, F. M. Holly, A. Verwey. Practical aspects of computational river hydraulics. Vol 3 of Monographs and surveys in water resources engineering, Pitman, London, 1980.

[5] Y. Ding, Y. Jia, S.Y. Wang. Identification of Manning's roughness coefficients in Shallow Water flows. Journal of Hydraulic Engineering, 130 (6) pp. 501-510, 2004.

[6] E. D. Fernandez-Nieto, D. Bresch, J. Monnier A consistent intermediate wave speed for a well-balanced $H L L C$ solver C. R. Acad. Sci. Paris, Ser. I 346, pp 795-800, 2008.

[7] E. Fernandez-Nieto, J. Marin, J. Monnier. Superposing 1D-2D shallow water models : source terms and finite volume schemes. Submitted.

[8] I. Y. Gejadze, J. Monnier. On a 2D zoom for 1D shallow-water model : coupling and data assimilation. Comp. Meth. Appl. Mech. Eng. Vol. 196, issues 45-48, pp 4628-4643, 2007.

[9] N. Goutal, F. Maurel. A finite volume solver for 1D shallow-water equations applied to an actual river. Int. J. Numer. Meth. Fluids, vol 38, pp 1-19, 2002.

[10] M. Greco, E. Oliveri, A. Leopardi, Two-Dimensional Modeling of Floods to Map Risk-Prone Areas. J. Water Resour. Plng. and Mgmt., Vol 128, Issue 3, pp 168-178, 2002.

[11] DassFlow software. DassFlow : Data Assimilation for free surface Flows. http ://www-gmm.insa-toulouse.fr/ monnier/DassFlow

[12] M. Honnorat, J. Marin, J. Monnier, X. Lai. Dassflow v1.0 : a variational data assimilation software for $2 D$ river flows. INRIA Research Report number 6150, 2007. 
[13] M. Honnorat, J. Monnier, FX Le Dimet. Lagrangian data assimilation for river hydraulics simulations. Comput. Visu. Sc. (Published on-line in march 2008). Vol. 12, Issue 5, pp. 235, 2009.

[14] M. Honnorat, J. Monnier, N. Rivière, E. Huot, FX Le Dimet. Identification of equivalent topography in an open channel flow using Lagrangian data assimilation. Comput. Visu. Sc. (Published on-line in 2009). In press.

[15] R. Hostache, X. Lai, J. Monnier, C. Puech. Assimilation of spatial distributed water levels into a shallow-water flood model. Part II : use of a remote sensing image of Mosel river. J. Hydrology. Submitted.

[16] X. Lai, J. Monnier. Assimilation of spatial distributed water levels into a shallow-water flood model. Part I : mathematical method and toy test case. J. Hydrology. Published on-line July'09. In press.

[17] E. Miglio, S. Perotto, F. Saleri. Model coupling techniques for free-surface flow problems : Part I. Nonlinear Analysis, vol 63, pp 1885-1896, 2005.

[18] H. Roux. Estimation de paramètres en hydraulique fluviale, à partir de données caractéristiques de l'imagerie aérienne. $\mathrm{PhD}$ Thesis, Institut National Polytechnique de Toulouse, 2004.

[19] B. Smith, P. Bjorstad, W. Gropp. Domain Decomposition. Cambridge University Press, 1996.

[20] G. Steinebach, S. Rademacher, P. Rentrop, M. Schulz. Mechanisms of coupling in river flow simulation systems. J. Comp. Appl. Math., vol 168, pp 459-470, 2004.

[21] E F. Toro. Shock-Capturing Methods for Free-Surface Shalow Flows. Wiley and Sons, Ltd, 2001. 